Article

\title{
Congestion Risk-Averse Stochastic Unit Commitment with Transmission Reserves in Wind-Thermal Power Systems
}

\author{
Yu Huang ${ }^{1}$, Qingshan $X u^{1, *}$ and Guang Lin ${ }^{2, *}$ \\ 1 School of Electrical Engineering, Southeast University, Nanjing 210096, China; huangyu@seu.edu.cn \\ 2 Department of Mathematics, Purdue University, West Lafayette, IN 47907, USA \\ * Correspondence: xuqingshan@seu.edu.cn (Q.X.); guanglin@purdue.edu (G.L.); \\ Tel.: +86-13813837607 (Q.X.); +1-765 4941965 (G.L.)
}

Received: 13 August 2018; Accepted: 18 September 2018; Published: 22 September 2018 updates

Featured Application: We present a congestion risk-averse stochastic unit commitment model with transmission reserves to hedge against uncertainty and variability induced by wind generation; the transmission reserve requirements are quantified in a systematic manner with relatively small computational effort and high accuracy; the proposed method can flexibly manage the uncertainty-induced congestion to enhance the system reliability and wind power utilization.

\begin{abstract}
The great proliferation of wind power generation has brought about great challenges to power system operations. To mitigate the ramifications of wind power uncertainty on operational reliability, predictive scheduling of generation and transmission resources is required in the day-ahead and real-time markets. In this regard, this paper presents a risk-averse stochastic unit commitment model that incorporates transmission reserves to flexibly manage uncertainty-induced congestion. In this two-settlement market framework, the key statistical features of line flows are extracted using a high-dimensional probabilistic collocation method in the real-time dispatch, for which the spatial correlation between wind farms is also considered. These features are then used to quantify transmission reserve requirements in the transmission constraints at the day-ahead stage. Comparative studies on the IEEE 57-bus system demonstrate that the proposed method outperforms the conventional unit commitment (UC) to enhance the system reliability with wind power integration while leading to more cost-effective operations.
\end{abstract}

Keywords: stochastic programming; unit commitment; transmission reserves; wind power forecast; real-time dispatch; uncertainty quantification

\section{Introduction}

The emerging trend of wind power integration into bulk power systems has raised great concern worldwide over the past decade [1]. Although wind generation is regarded as an eco-friendly energy resource with zero marginal cost, the uncertain and intermittent nature of wind power has posed grand challenges to utility operators in terms of keeping the system reliable and cost-effective [2]. More specifically, the network under high wind penetration is prone to congestion issues, i.e., large fluctuations of line flows due to inherent wind power uncertainty, making it difficult to promote the deployment of wind energy in the grid. The undesired consequences of congestion such as generation reserve undeliverability, wind curtailment, and load shedding would add extra operational costs, diminish security margins, or even further demoralize investment in the wind industry. Therefore, there is a need for methods that allow an effective utilization of the increasing 
wind generation when faced with transmission congestion, as well as other technical limits of thermal generators (e.g., capacity limits, ramping limits).

The existing strategies that are applied to alleviate the uncertainty-induced congestion can be categorized into two groups: improving the accuracy of wind power forecasting as well as modeling on the wind farm side, and providing more operational flexibility from the power system side.

It is generally acknowledged that forecast accuracy deteriorates as the time horizon grows. Specifically, the error for a day-ahead forecast can vary from $15-25 \%$ using the current NWP-based approach $[3,4]$, and this value could be even higher during the period of extreme events such as unexpected wind power ramps [5]. The forecast uncertainty is taken into account by modeling the forecast errors statistically with various types of distributions (e.g., Gaussian [6], Weibull [7] or Beta distributions [8]). More deep research has found that forecast errors are conditional to the given point forecasts, and thus, the error distribution should be versatile with respect to different forecast levels [8]. In [9], copula theory is employed to depict the spatial correlation of forecast errors among different wind farms in a neighboring area. Pinson et al. [10] argue that half of the extra cost due to the inaccuracy of forecast could be saved if forecast errors are properly considered in a liberalized electricity market.

As for the latter category, we seek to come up with solutions in a systematic manner relying on either infrastructural supplements (e.g., energy storage systems (ESS) [11], demand response (DR) resources [12], and flexible alternating current (AC) transmission systems (FACTS) [13]), or enhancement of utilization of the existing infrastructure and methodology framework. The formal effort is straightforward and effective in mitigating wind power uncertainty; however, it requires a huge amount of capital investment, as well as other operation and maintenance costs relating to the equipment [14]. To circumvent such a problem, a less expensive approach to congestion management is achieved by modifying the network topology with transmission switching [15]. In [16], different types of DR programs are explored to evaluate their impacts on the reliability of the wind integrated power system. Scheduling adequate generation spinning reserves is the most commonly-used way of providing the required flexibility to hedge against the risk of unexpected real-time branch congestion [17]. Then, the question of how to quantify the reserve capacity to avoid assuming a fixed reserve rate arises naturally, as in the traditional deterministic framework [18]. Reference [19] presents a dynamic reserve scheduling approach on an hourly basis according to the realistic wind power conditions, and the results are compared with the static reserve method. The work presented in [20] solves this problem in a scenario-based stochastic unit commitment (SUC) formulation, which uses a number of possible scenarios to cover a credible range of the future wind power availability. However, it is time-consuming to run the crude Monte Carlo simulation (MCS) for all input scenarios, especially when high computation accuracy is needed. Robust optimization is employed in [21] solely for the worst-case scenario, which may lead to highly conservative results that are far from reality. Another critical concern of the committed generation reserve is whether it is deliverable when needed. When the generation reserve is undeliverable due to the binding power flow constraints, operators may resort to wind curtailment and load shedding [22]. To address this issue, a risk-based constraint could be embedded in each line based on the line flows calculated via the probabilistic optimal power flow (P-OPF) [23,24]. Reference [25] proposes a power system partitioning method to commit the reserves locally in the accrued zones by minimizing the risk of intra-zonal transmission congestion. In the resent study [26], transmission reserves have been introduced and have served as a complementary tool in case of generation reserve undeliverability.

In this paper, with the goal of limiting the risk of uncertainty-induced transmission congestion and reserve undeliverability, we propose a risk-averse predictive method for dynamic transmission reserves in a SUC framework. The proposed model is formulated as a bi-level optimization which can be decomposed into day-ahead unit commitment (UC) and probabilistic real-time redispatch. The redispatch problem in the lower level is solved to capture the statistical features of line flows using a high-dimensional probabilistic collocation method (PCM) with correlation control. The results are 
then used to quantify the predictive transmission reserve requirements as a constraint of UC in the upper level. In summary, the main contributions of our work can be outlined as follows:

- The risk of transmission congestion is formulated in the form of predictive transmission reserves, which is incorporated in the SUC framework to improve the operational flexibility and capability of facing unexpected wind power realizations.

- The transmission reserve requirements are dynamically quantified in the UC based on the probabilistic real-time line flow information, rather than using the deterministic way of assigning a predefined portion of total transmission capacity.

- The statistical features of line flows in response to the multi-correlated wind power uncertainty are systematically characterized in a probabilistic redispatch problem using the high-dimensional PCM, which avoids the complexity induced by numerous scenarios in the traditional MCS-based approaches.

\section{Problem Formulation}

\subsection{Modeling Conditional Forecast Error for Multiple Wind Farms}

The forecast uncertainty of wind power is characterized by probabilistic modeling of the forecast error with continuous probability density function (PDF). In most studies, the forecast error is simply represented by Gaussian distribution with zero mean and wind power standard deviation $\sigma_{W}$. However, the Gaussian assumption has known limitations as fixed kurtosis $(\kappa=3)$ which is inappropriate for fitting the skewed and fat-tailed error distribution $(3<\kappa<10)$ [8]. Moreover, the forecast error distribution is a conditional PDF with respect to the day-ahead point forecast, showing significant diversity in different forecast levels [27]. Therefore, we adopt the following procedure [8] to generate the PDF of wind power output for a given forecast using the actual wind data. First, the standardized forecast range $([0,1]$ p. u.) is evenly divided into several levels, namely the forecast bins. Then, the data pairs [actual forecast] of wind power at each time step are sorted by the point forecast and assigned to the corresponding forecast bins. Finally, the specific distribution is selected to fit the actual wind power PDF at each forecast bin. The beta distribution is employed here because it has variable skewness and kurtosis, and its value is limited to the interval $[0,1]$ which matches the range of normalized wind power.

The way of modeling the dependence of adjacent wind farms is through the correlation coefficient obtained from numerous historical wind power measurement data, which is expressed as

$$
\rho_{i j}=\frac{\operatorname{Cov}\left(W_{i}, W_{j}\right)}{\sigma_{i} \sigma_{j}}=\frac{\sum_{t \in \mathcal{T}}\left(W_{i}^{t}-\bar{W}_{i}\right)\left(W_{j}^{t}-\bar{W}_{j}\right)}{\sqrt{\sum_{t \in \mathcal{T}}\left(W_{i}^{t}-\bar{W}_{i}\right)^{2}} \sqrt{\sum_{t \in \mathcal{T}}\left(W_{j}^{t}-\bar{W}_{j}\right)^{2}}}
$$

where $\operatorname{Cov}(\cdot)$ denotes the covariance operator; $W_{i}^{t}$ and $W_{j}^{t}$ are actual wind power at bus $i, j$ and time $t$; $\bar{W}_{i}$ and $\bar{W}_{j}$ are the means of wind power at bus $i, j$ throughout the whole time periods, and $\sigma_{i}, \sigma_{j}$ are their standard deviations. The correlation coefficient is 1 indicating a perfect linear relationship, and is 0 in case of uncorrelation. Then the coefficient $\rho$ makes up of the correlation coefficient matrix $\boldsymbol{R}_{W}$ for $n$ wind farms, defined as

$$
\boldsymbol{R}_{W}=\left(\begin{array}{ccc}
1 & \cdots & \rho_{1 n} \\
\vdots & \ddots & \vdots \\
\rho_{n 1} & \cdots & 1
\end{array}\right)_{n \times n}, \rho_{i j}=\rho_{j i}
$$

It is noted that $\boldsymbol{R}_{W}$ only measures the degree of linear dependence among wind farms, and should be modified when undergoing non-linear transformations (e.g., Nataf transformation [28]). 


\subsection{Risk Averse Stochastic Unit Commitment with Transmission Reserves}

In this section, we present a risk-averse SUC model with a predictive method for transmission reserves to enhance the utilization of the available wind power and alleviate the transmission congestion in the grid. The proposed framework is formed as a bi-level problem on the typical two-stage market basis, i.e., the day-ahead and real-time market. The upper-level problem is the day-ahead UC, where the objective is to decide the day-ahead status of the thermal generation units, including unit on/off status and amount of power generated at each time period, while the unit and system constraints involving the transmission congestion limit are satisfied. The objective function of UC $f_{1}\left(x_{U C}\right)$ is to minimize the total operation cost, which consists of the no-load cost, start-up cost, spinning reserve cost and variable cost of all generators. The lower level problem is the real-time economic dispatch (ED) process with remedial measures under the wind power uncertainty, and its objective function $f_{2}\left(x_{E D}\right)$ is the expected redispatch cost which includes the cost of real-time dispatch and remedial measures (wind curtailment and load shedding). The main purpose of the ED sub-problem is to obtain the statistical features of line flows for the estimation of the upper bound of transmission reserve in the upper level. Since the two objectives in both levels are of the same type (the operation cost of generators), we can simply combine them as a unified one, which is written as

$$
\operatorname{Min} f_{1}\left(x_{U C}\right)+f_{2}\left(x_{E D}\right)
$$

The two items of the objective function are detailed as

$$
\begin{aligned}
& f_{1}\left(x_{U C}\right)=\sum_{t \in \mathcal{T}} \sum_{g \in \mathcal{G}}\left(C_{g} P_{g}^{t}+S U C_{g} u_{g}^{t}+N C_{g} x_{g}^{t}+R C_{g} r_{g}^{t}\right), \\
& f_{2}\left(x_{E D}\right)=\sum_{t \in \mathcal{T}} \sum_{g \in \mathcal{G}} C_{g} \hat{P}_{g}^{t}+\sum_{t \in \mathcal{T}} \sum_{n \in \mathcal{N}}\left(C_{c r} W_{n, c r}^{t}+C_{l s} D_{n, l s}^{t}\right)
\end{aligned}
$$

where $P_{g}^{t}, u_{g}^{t}, x_{g}^{t}$, and $r_{g}^{t}$ are, respectively, the dispatch, start-up, on/off and generation spinning reserve decisions of generator $i$ at time $t$, and $C_{g}, S U C_{g}, N C_{g}$ and $R C_{g}$ are their associated cost coefficients, respectively. $\hat{P}_{g}^{t}$ is the actual power production at time $t$ in the real-time dispatch. The penalty cost terms of wind curtailment $W_{n, c r}^{t}$ and load shedding $D_{n, l s}^{t}$ are proportional to the Value of Wind Curtailment (VOWC) $C_{c r}$ and Value of Lost Load (VOLL) $C_{l s}$, respectively.

The constraints of the aforementioned SUC model are given as follows:

- The Unit Constraints:

$$
\begin{gathered}
x_{g}^{t}, u_{g}^{t}, v_{g}^{t} \in\{0,1\}, t \in \mathcal{T}, g \in \mathcal{G} \\
x_{g}^{t}-x_{g}^{t-1}=u_{g}^{t}-v_{g}^{t}, t \in \mathcal{T}, g \in \mathcal{G} \\
\sum_{\tau=t-U T_{g}+1}^{t} u_{g}^{\tau} \leq x_{g}^{t}, t \in \mathcal{T}, g \in \mathcal{G} \\
\sum_{\tau=t-D T_{g}+1}^{t} v_{g}^{\tau} \leq 1-x_{g}^{t}, t \in \mathcal{T}, g \in \mathcal{G} \\
P_{g}^{\min } x_{g}^{t} \leq P_{g}^{t} \leq\left(P_{g}^{\max }-r_{g}^{t}\right) x_{g}^{t}, t \in \mathcal{T}, g \in \mathcal{G} \\
-R_{g}^{D} \leq P_{g}^{t}-P_{g}^{t-1} \leq R_{g}^{U}, t \in \mathcal{T}, g \in \mathcal{G} \\
r_{g}^{t} \leq R_{g}^{10} x_{g}^{t}, t \in \mathcal{T}, g \in \mathcal{G}
\end{gathered}
$$

- The System Constraints:

$$
\begin{gathered}
\sum_{g \in \mathcal{G}} P_{g}^{t}=\sum_{n \in \mathcal{N}}\left(D_{n}^{t}-\hat{W}_{n}^{t}\right), t \in \mathcal{T} \\
\left|P_{l}^{t}\right|=\left|\sum_{n \in \mathcal{N}} S_{l, n}\left(D_{n}^{t}-\hat{W}_{n}^{t}-\sum_{g \in \mathcal{G}_{n}} P_{g}^{t}\right)\right| \leq P_{l}^{\max }, t \in \mathcal{T}, l \in \mathcal{L}
\end{gathered}
$$




$$
\sum_{g \in \mathcal{G}} r_{g}^{t} \geq \sum_{n \in \mathcal{N}}\left(\gamma_{D} D_{n}^{t}+\gamma_{W} \hat{W}_{n}^{t}\right), t \in \mathcal{T}
$$

where $G, N, L, T$ are the sets of generators, nodes, transmission lines and time periods, respectively; $G_{n} \subseteq G$ is the subset of generators at bus $n$; $v_{g}^{t}$ is the shut-down binary variable of generator $g$ at time $t ; P_{g}^{\min }$ and $P_{g}^{\max }$ are the minimum and maximum output levels of generator $g ; R_{g}^{D}$ and $R_{g}^{U}$ are the ramp-down and ramp-up rates of generator $g$, and $R_{g}^{10}$ is the 10-min ramp rate. $D T_{g}$ and $U T_{g}$ are the minimum-down/ -up times of generator $g . P_{l}^{\max }$ is the power flow limit for line $l . D_{n}^{t}$ and $\hat{W}_{n}^{t}$ denote the net load and day-ahead wind power forecast at bus $n$ and time $t$, respectively, with the reserve capacity coefficients $\gamma_{D}$ and $\gamma_{W} \cdot S_{l, n}$ is the shift factor which characterizes the contribution from bus $n$ to power flow on line $l$.

Equations (5) and (6) correspond to units on/off, start-up and shut-down constraints. Equations (7) and (8) are the constraints of the generator's minimum up and downtime. Equation (9) enforces the maximum and minimum generation capacity limits. Equation (10) enforces ramping up and down limits. Equation (11) confines the reserve capacity to the 10-min generation ramping capability. As for the system constraints, Equation (12) is the power balance constraint, which ensures that the power provided by generators must be equal to the net-demand (load minus the wind power) at all times. Equation (13) applies the DC power flow model to impose transmission line limits. Equation (14) establishes the minimum system-wide reserve requirements.

The aforementioned constraints (Equations (5)-(14)) are common in conventional UC formulations. In this work, we extend to quantify the transmission reserve requirements in the UC by imposing a limit on the risk of congestion in real-time dispatch. According to Cantelli's inequality [29], the upper bound of congestion probability on line $l$ is formed as

$$
\operatorname{Pr}\left(P_{l}^{t}>P_{l}^{\max }\right)=\operatorname{Pr}\left(P_{l}^{t}-\bar{P}_{l}^{t}>P_{l}^{\max }-\bar{P}_{l}^{t}\right) \leq \Pi_{\max }
$$

where

$$
\Pi_{\max }=\frac{\sigma_{P_{l}^{t}}^{2}}{\sigma_{P_{l}^{t}}^{2}+\left(P_{l}^{\max }-\bar{P}_{l}^{t}\right)^{2}}, \Pi_{\max } \leq \alpha_{l}
$$

where $\alpha_{l}$ is a user-defined parameter, called Conservativeness Factor, which is based on the degree of conservativeness in assessing the risk of congestion. This parameter is similar to the confidence level defined in the chance-constraint programming [30] that enforces the inequality to a certain level of probability; thus, $0<\alpha_{l}<1$. Equation (16) can be rewritten in the following form of constraint:

$$
\left|\bar{P}_{l}^{t}\right| \leq P_{l}^{\max }-\sigma_{P_{l}^{t}} \sqrt{\frac{1}{\alpha_{l}}-1}, t \in \mathcal{T}, l \in \mathcal{L}
$$

Rearranging Equation (17) to get rid of the sign of absolute value, yields:

$$
-P_{l}^{\max }+\Gamma_{l}^{t} \leq P_{l}^{t} \leq P_{l}^{\max }-\Gamma_{l}^{t}, t \in \mathcal{T}, l \in \mathcal{L}
$$

where

$$
\Gamma_{l}^{t}=\left\{\begin{array}{l}
\bar{P}_{l}^{t}-P_{l}^{t}+\sigma_{P_{l}^{t}} \sqrt{1 / \alpha_{l}-1}, P_{l}^{t} \geq 0 \\
P_{l}^{t}-\bar{P}_{l}^{t}+\sigma_{P_{l}^{t}} \sqrt{1 / \alpha_{l}-1}, P_{l}^{t}<0
\end{array}\right.
$$

It should be mentioned that the constraint in Equation (18) is comparable to the original transmission line constraint in Equation (13), with an additional term $\Gamma_{l}^{t}$. Therefore, $\Gamma_{l}^{t}$ can be interpreted as the required transmission reserve for line $l$ at time $t$. In other words, if $\Gamma_{l}^{t}$ for each line and time step is scheduled in day-ahead UC as a portion of the transmission capacity in (19), it is assured that the probability of congestion will not be larger than $\alpha_{l}$ and the system will be safe and reliable under wind power uncertainty. 
To implement the conditional constraints in Equation (19), which depends on the direction of the line flow, it is necessary to apply the strategy in [31] to reformulate it as a number of linear constraints involving auxiliary variables.

\section{Quantifying Transmission Reserve Requirements in Real-Time Dispatch}

\subsection{Basic Real-Time Dispatch Model with Corrective Measures}

As is observed in the previous section, the congestion constraint (18) in the risk-averse SUC formulation proposed is dependent on the key statistical features of the line flows in real-time operation, i.e., the mean and standard deviation of line flows, $\bar{P}_{l}^{t}$ and $\sigma_{P_{l}^{t}}$, respectively, which can be obtained by solving the P-OPF sub-problem. The uncertainties in the problem are generally represented by a set of scenarios $s \in \mathcal{S}$ using the crude MCS, where the two key statistics of line flows are expressed as

$$
\begin{gathered}
\bar{P}_{l}^{t}=\frac{1}{N_{s}} \sum_{s \in \mathcal{S}} \hat{P}_{l}^{t, s} \\
\sigma_{P_{l}^{t}}^{2}=\frac{1}{N_{s}-1} \sum_{s \in \mathcal{S}}\left(\hat{P}_{l}^{t, s}\right)^{2}-\left(\bar{P}_{l}^{t}\right)^{2}
\end{gathered}
$$

where $N_{s}$ is the number of Monte Carlo (MC) scenarios of set $S$. The superscript $s$ denotes the index for the scenario of random variables, $s=1,2, \cdots, N_{s}$. Other constraints in the real-time dispatch model are given as follows:

$$
\begin{gathered}
P_{g}^{\min } x_{g}^{t} \leq \hat{P}_{g}^{t, s} \leq P_{g}^{\max } x_{g}^{t}, g \in \mathcal{G}, t \in \mathcal{T}, s \in \mathcal{S} \\
\sum_{g \in \mathcal{G}} \hat{P}_{g}^{t, s}=\sum_{n \in \mathcal{N}}\left(\hat{W}_{n}^{t, s}-W_{n, c r}^{t, s}-D_{n}^{t, s}+D_{n, l s}^{t, s}\right), t \in \mathcal{T}, s \in \mathcal{S} \\
\hat{P}_{l}^{t, s}=\sum_{n \in \mathcal{N}} S_{l, n}\left(\sum_{g \in \mathcal{G}_{n}} \hat{P}_{g}^{t, s}+\hat{W}_{n}^{t, s}-W_{n, c r}^{t, s}-D_{n}^{t, s}+D_{n, l s}^{t, s}\right), t \in \mathcal{T}, l \in \mathcal{L} \\
-P_{l}^{\max } \leq \hat{P}_{l}^{t, s} \leq P_{l}^{\max }, l \in \mathcal{L}, t \in \mathcal{T}, s \in \mathcal{S} \\
W_{n, c r}^{t, s} \geq 0, D_{n, l s}^{t, s} \geq 0, n \in \mathcal{N}, t \in \mathcal{T}, s \in \mathcal{S}
\end{gathered}
$$

Equations (22)-(26) represent, respectively, the generation capacity, power balance and line flow limits in the real-time dispatch, with the consideration of remedial measures including wind curtailment and load shedding. The two remedies used are incorporated in both the constraints and the objective function of the real-time dispatch to recover the solution from infeasibility, which is a main difference from the conventional P-OPF. The procedure of conducting the proposed risk-averse SUC is depicted with the flowchart shown in Figure 1.

However, the execution time involved to conduct the above optimization problem could be undoubtedly large, due to a significant number of MC scenarios required to reach an acceptable accuracy. A more computationally efficient method, namely PCM, is developed herein, and its extended version that is adaptable to high-dimensional and correlated cases will be covered in the following subsection. 


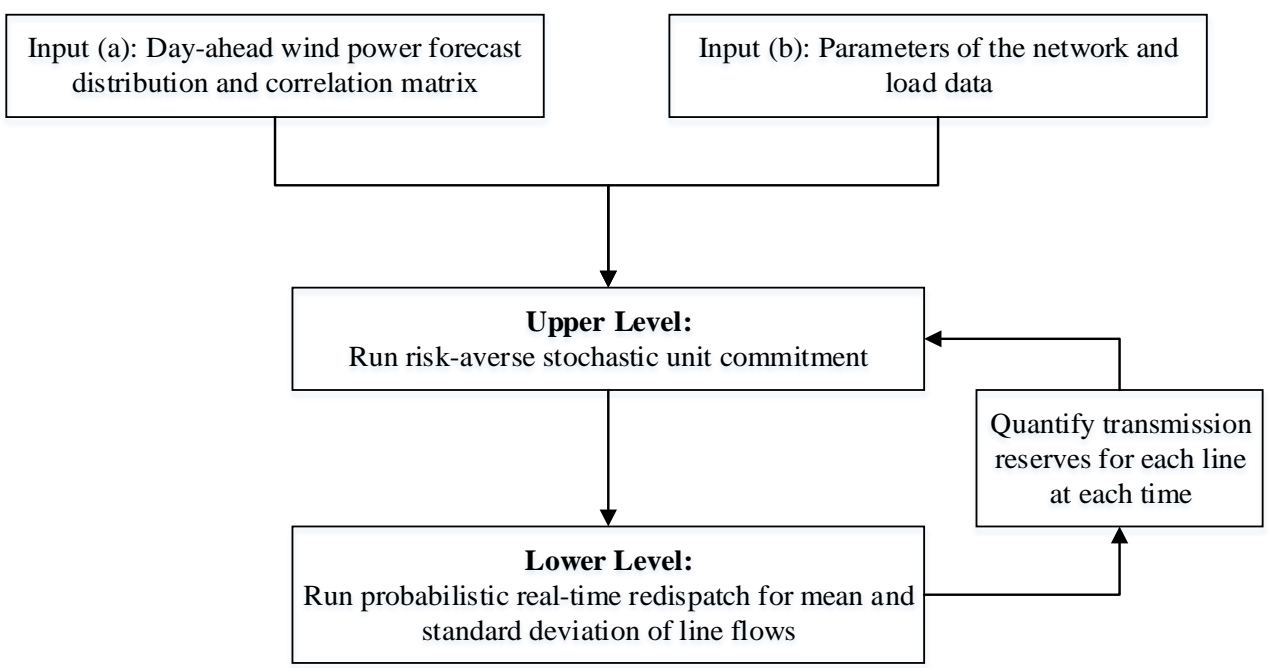

Figure 1. Flowchart of the proposed risk-averse stochastic unit commitment (SUC) with transmission reserves.

\subsection{Probabilistic Collocation Method with Correlation Control}

The PCM is used to quantify the statistical features of the uncertain line flows in the probabilistic real-time dispatch sub-problem. This method is efficient for handling uncertainties with a much smaller number of selected scenarios, i.e., collocation points (CPs), than what is required from the traditional random sampling techniques (e.g., MCS), and is considered an extension of the generalized point estimate method (PEM) [32]. Fully explained in [33], the CPs selected are based on Gaussian quadrature (GQ) rule that could reach an algebraic accuracy of $2 N-1$ by $N$ CPs. Furthermore, a simple correlation control technique is also combined with the basic PCM to deal with the correlation between input variables, i.e., wind power outputs of multiple wind farms. The procedure of evaluating the mean and standard deviation of the random line flows is summarized as follows:

(1) Suppose $\xi_{n}, \forall n \in \mathcal{N}$ is a standard normal vector. Generate $N_{C} C P s$ and their corresponding weights of $\xi_{n}$ using the Multiprecision Computing Toolbox [34]. The sth CP is denoted as $\xi_{n}^{s}, s \in \mathcal{S}=$ $\left\{1,2, \cdots N_{C}\right\}, \forall n \in \mathcal{N}$, whose elements are independent of each other.

(2) Transform the $\mathrm{CP} \xi_{n}^{s}$ to the correlated ones $z_{n}^{s}$ by

$$
\boldsymbol{z}_{n}^{s}=L \boldsymbol{\xi}_{n}^{s}, s \in \mathcal{S}, \forall n \in \mathcal{N}
$$

where $L$ is a lower triangular matrix, obtained by Cholesky decomposition of the correlation coefficient matrix $\boldsymbol{R}_{W}$

$$
\boldsymbol{R}_{W}=\boldsymbol{L L}^{T}
$$

(3) Map each $z_{n}$ from the correlated standard normal domain into the original space for the input wind power $\hat{\mathrm{W}}_{n}$

$$
\hat{W}_{n}=F_{n}^{-1}\left(\Phi\left(z_{n}\right)\right), n \in \mathcal{N}
$$

where $\Phi$ and $F_{n}$ are the cumulative probability function (CDF) of $z_{n}$ and $\hat{W}_{n}$, respectively. $F_{n}^{-1}$ is the inverse CDF of $\hat{W}_{n}$. Thus, the CPs of $\hat{W}_{n}$ at each time step $t$ is obtained and denoted as $\hat{W}_{n}^{t, s}$ with the corresponding weight $\omega_{s}$.

(4) Solve the deterministic optimization problem of real-time dispatch at each wind power scenario $\hat{W}_{n}^{t, s}$ to yield the associated output scenario, i.e., $\hat{P}_{g}^{t, s}$ and $\hat{P}_{l}^{t, s}$, and then substitute Equations (20) and (21) for a new expression of the line flow statistics

$$
\bar{P}_{l}^{t}=\sum_{s \in \mathcal{S}} \omega_{s} \hat{P}_{l}^{t, s}, t \in \mathcal{T}, l \in \mathcal{L}
$$




$$
\sigma_{P_{l}^{t}}^{2}=\sum_{s \in \mathcal{S}} \omega_{s}\left(\hat{P}_{l}^{t, s}\right)^{2}-\left(\bar{P}_{l}^{t}\right)^{2}, t \in \mathcal{T}, l \in \mathcal{L}
$$

\subsection{High Dimensionality and Sparse Grids}

Since GQ rule achieves high accuracy for one-dimensional approximation, it is natural to extend it to multivariate cases where there are multiple random variables. The most straightforward way is through tensor product approach. Let $\Theta_{d}$ be a $d$-dimensional CP set, which can be constructed by tensor product of $\Theta_{1}$ at each dimension as

$$
\Theta_{d}=\Theta_{1}^{m_{1}} \times \cdots \times \Theta_{1}^{m_{d}}
$$

where the superscript $m_{i}$ denotes the number of CPs based on GQ rule at dimension $i$. Assume that $m_{1}=\cdots=m_{d}=m$, then it requires solving the real-time dispatch problem $m^{d}$ times, which is obviously intractable for large dimensions. To tackle this issue, Smolyak sparse grid [35] is employed which is expressed as a linear combination of the tensor product

$$
\Theta_{d}=\underset{N-d+1 \leq|\mathbf{m}| \leq N}{\cup}\left(\Theta_{1}^{m_{1}} \times \cdots \times \Theta_{1}^{m_{d}}\right)
$$

where $|\boldsymbol{m}|=\sum_{i=1}^{d} m_{i} ; N \geq d$ is an integer denoting the construction level. It is clear that the resulting grid set is a union of the collection of subsets of the full tensor grids. Hence, the curse of dimensionality, albeit still exist, is largely lessened. An example of the comparison between two-dimensional sparse grids and tensor product is shown in Figure 2, where a significant reduction in the number of CPs is observed.

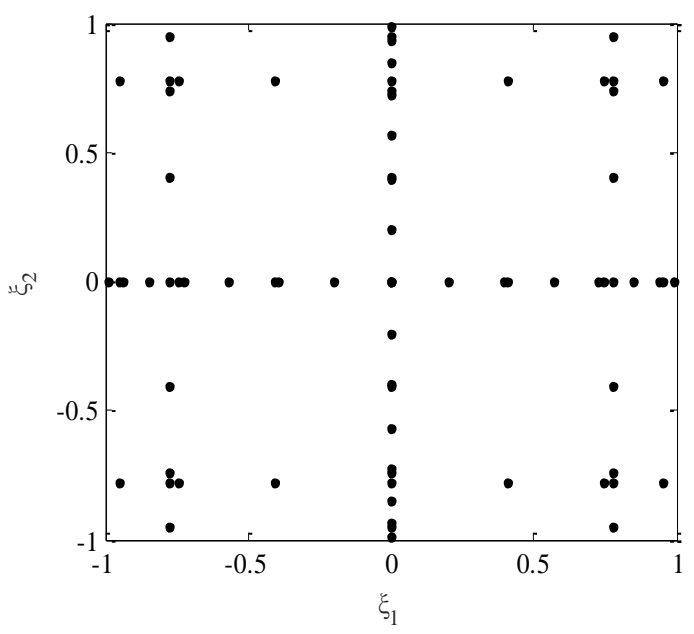

(a)

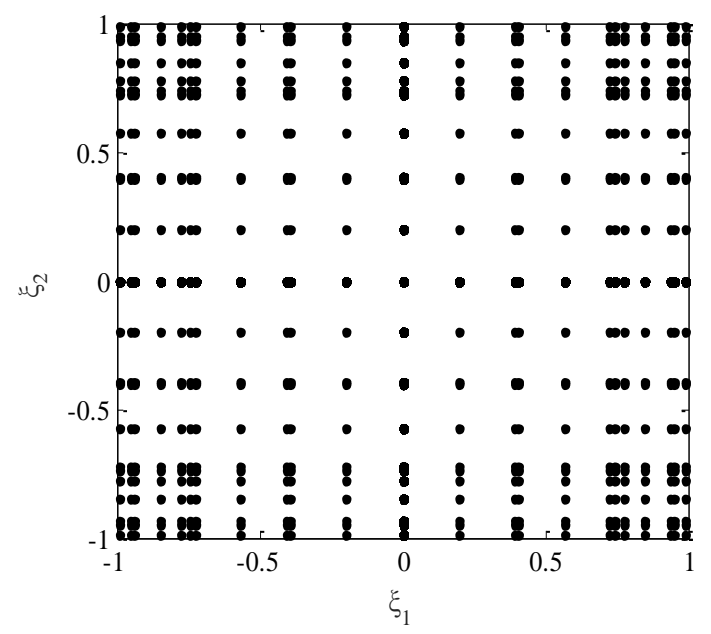

(b)

Figure 2. Two-dimensional collocation points (CPs) based on Gaussian quadrature (GQ) rule at level 3: (a) Smolyak sparse grids. The total number of CPs is 75; (b) Tensor product grids. The total number of CPs is 529 .

\section{Discussion}

Comparing the transmission line constraint in Equation (16) of the proposed risk-averse SUC with Equation (13) as in the conventional UC, we can see that a portion of the total transmission capacity, defined as a new concept of predictive transmission reserves, is maintained by considering the risk of wind power uncertainty-induced congestion. A highly desirable feature of this inequality is that it derives from the chance-constraint in Equation (15) but has a linear form. Therefore, the chanced-constraint SUC framework is recasted as a mixed integer programming (MIP) problem which 
can be solved by most commercially available solvers instead of using heuristic methods. In [36], a pre-computed transmission reliability margin is assigned to each line to accommodate various uncertainties using the basic statistical and network information. However, the impact of real-time redispatch on the line flows is not considered, whereas the quantification of transmission reserves in our work is adaptive to the current operating conditions.

To the best of our knowledge, this is the first time in the literature where the SUC with a predictive model for transmission reserves is formulated, and the reserve requirements are quantified in such a precise manner. While several limitations still exist in the current work that worth further investigating. Firstly, in addition to the wind power uncertainty, other uncertainty sources including the credible $N-1$ outages of lines and components should also be considered which directly influence the mean and standard deviation of the line flows, as estimated in the real-time redispatch. Secondly, it is worth developing a more comprehensive probabilistic wind power forecast model that accounts for both spatial and temporal correlations between the wind power scenarios. Finally, the Smolyak spares grid used for scenario reduction could be coupled with parallel computing technique [23] to cope with more complex power systems of higher dimensionality.

\section{Case Study}

The performance of the proposed day-ahead risk averse stochastic unit commitment (RA-SUC) model with transmission reserves is tested on a modified IEEE 57-bus system, with 6 wind farms located at buses 30, 31, 32, 33, 36 and 40. The wind power output and the forecast data are obtained from State Grid Electric Power Research Institute [37] database in Nantong grid of total 365 days of the year 2017, each with $100 \mathrm{MW}$ nominal capacity. The forecast uncertainty is assumed to be conditional to the day-ahead point forecast and follow a beta distribution characterized by the correlation coefficient matrix. The load data and other parameters of the network are taken from [38] and remain unchanged. To observe more number of incidents of congestion and verify the effectiveness of the proposed method in alleviating the congestion, all the line capacities are lowered to half of their nominal ratings. Other parameters in the model are given as VOLL $=2000 \$ / \mathrm{MWh}, \mathrm{VOWC}=50 \$ / \mathrm{MWh}$, $\gamma_{D}=\gamma_{W}=5 \%$. The study is implemented in MATLAB 2014b (MathWorks, Natick, MA, USA) and ILOG CPLEX 12.6 (IBM, Armonk, NY, USA, 2015) with its default settings [39] on a personal computer (PC) with Intel Core i5 $3.0 \mathrm{GHz}$ and 3GB RAM. Four different cases are carried out to illustrate the impact of wind power penetration $P_{W}$ and congestion conservativeness factor $\alpha_{l}$ (Case 1: $P_{W}=1$, $\alpha_{l}=0.2$; Case 2: $P_{W}=1, \alpha_{l}=0.45$; Case 3: $P_{W}=1.5, \alpha_{l}=0.2$; Case 4: $\left.P_{W}=1.5, \alpha_{l}=0.45\right)$ on the operation outcomes. The utility value of $P_{W}$ corresponds to the original wind power data and the value of 1.5 denotes that the wind power input is multiplied by 1.5 of the original data.

Table 1 lists the key expected economic indices obtained from RA-SUC and conventional UC (C-UC) with and without considering the wind power correlation. It is observed that the average operation cost by adopting RA-SUC is relatively lower than that of its counterpart C-UC for all four cases. In terms of wind utilization and load serve ratio, there is a slight increase in RA-SUC compared to C-UC as a result of the reduction of wind curtailment and load shedding. The ratios are close to the utility for case 2 and 4 , where there is a tighter acceptable risk of transmission congestion since $\alpha_{l}$ is larger. The proposed method also results in less required spinning reserves in real-time operation, thus improving the operational reliability of the system. Moreover, comparing the results on both sides of the table shows that the total cost and reserve requirements are intensified when accounting for wind power correlation. This observation is justified owing to the fact that when no correlation is considered, it is likely that the variations of wind power compensate for each other, leading to a smaller risk of transmission congestion. 
Table 1. Expected economic indices of the proposed method versus conventional unit commitment (UC). C-UC: conventional UC; RA-SUC: risk-averse stochastic unit commitment.

\begin{tabular}{cccccccccc}
\hline & & \multicolumn{3}{c}{ With Correlation } & \multicolumn{4}{c}{ Without Correlation } \\
\cline { 3 - 9 } & & Case 1 & Case 2 & Case 3 & Case 4 & Case 1 & Case 2 & Case 3 & Case 4 \\
\hline \multirow{2}{*}{ Total Cost [\$/day] } & RA-SUC & 53,784 & 45,748 & 52,574 & 45,983 & 52,313 & 45,022 & 49,468 & 45,537 \\
& C-UC & 55,652 & 49,294 & 56,620 & 47,125 & 53,659 & 48,356 & 54,532 & 46,749 \\
\hline Wind Utilization & RA-SUC & 88.41 & 93.74 & 90.53 & 96.93 & 89.28 & 93.82 & 90.47 & 98.10 \\
Ratio [\%] & C-UC & 87.67 & 91.93 & 89.70 & 93.71 & 87.91 & 92.13 & 89.78 & 95.58 \\
\hline Load Serve Ratio & RA-SUC & 95.26 & 98.21 & 94.87 & 99.83 & 95.24 & 98.21 & 95.18 & 99.85 \\
[\%] & C-UC & 95.12 & 98.26 & 94.57 & 99.36 & 95.21 & 98.15 & 94.59 & 99.69 \\
\hline Spinning Reserves & RA-SUC & 10,505 & 7186 & 9806 & 7458 & 11,002 & 7437 & 10,799 & 8062 \\
[MWh/day] & C-UC & 11,284 & 8849 & 10,892 & 9280 & 11,891 & 9166 & 11,680 & 9613 \\
\hline
\end{tabular}

In addition to the experiment results for the four specific scenarios in Table 1, Figures 3 and 4 compare the total operation cost and congestion hours of the proposed RA-SUC with C-UC under various $\alpha$ values for a range of wind power levels from 1 to 1.8. The black solid line corresponds to $\mathrm{C}-\mathrm{UC}$, whereas the other three dash-dot curves, which are well below the black one, corresponding to the proposed RA-SUC with three different $\alpha$ values $(\alpha=0.2,0.25$ and 0.3$)$. The significant reduction of operation cost and congestion hours under each wind power level confirms the effectiveness and robustness of the proposed method in enhancing the economic benefits and operation reliability. It is noted that assigning a larger $\alpha$ would increase the occurrence of congestion events as well as the total cost at high wind power levels. Specifically, in Figure 3, the operation cost decreases before it starts increasing at a certain wind power level ( $P_{W}=1.2$ or 1.3), regardless of whether the RA-SUC is used. Further, the proposed method has a much slower increasing rate of congestion hours compared to C-UC in Figure 4, indicating the improvement of system robustness to transmission congestion.

To interrogate the performance of high-dimensional PCM in solving the probabilistic real-time dispatch sub-problem, the obtained real-time transmission reserves using PCM with sparse grids (denoted by PCM-SG) are compared with those of 1000 MCS and two-point estimate method [32] (denoted by 2PEM) with regards to both accuracy and computational effort. Since these values vary for each time step and from line to line, we standardize the results by computing the average relative error and take the MCS with 20,000 scenarios as a benchmark. Figure 5 presents the average percentage error of real-time transmission reserve requirements for all the branches and cases over the whole time horizon. As can be seen, the PCM-SG consistently has the lowest percentage error for all the four cases with a maximum value of $6.3 \%$ in case 2 and a minimum value of $2.3 \%$ in case 3 . The relative errors of 2PEM in the four cases are kept almost invariant at around 10\%, showing less accuracy compared to PCM-SG, but are much lower than those of 1000 MCS, especially in case 1 and 3, where the error goes up to $20 \%$. The computational complexity of each method can be measured by the number of scenarios involved in the simulation. Though there are more scenarios needed in PCM-SG (109 scenarios) than 2PEM (13 scenarios), the reduction ratio is dramatic compared to 1000 MCS (183 vs 20), which is efficient for the optimization problem on the day-ahead and hourly basis. 


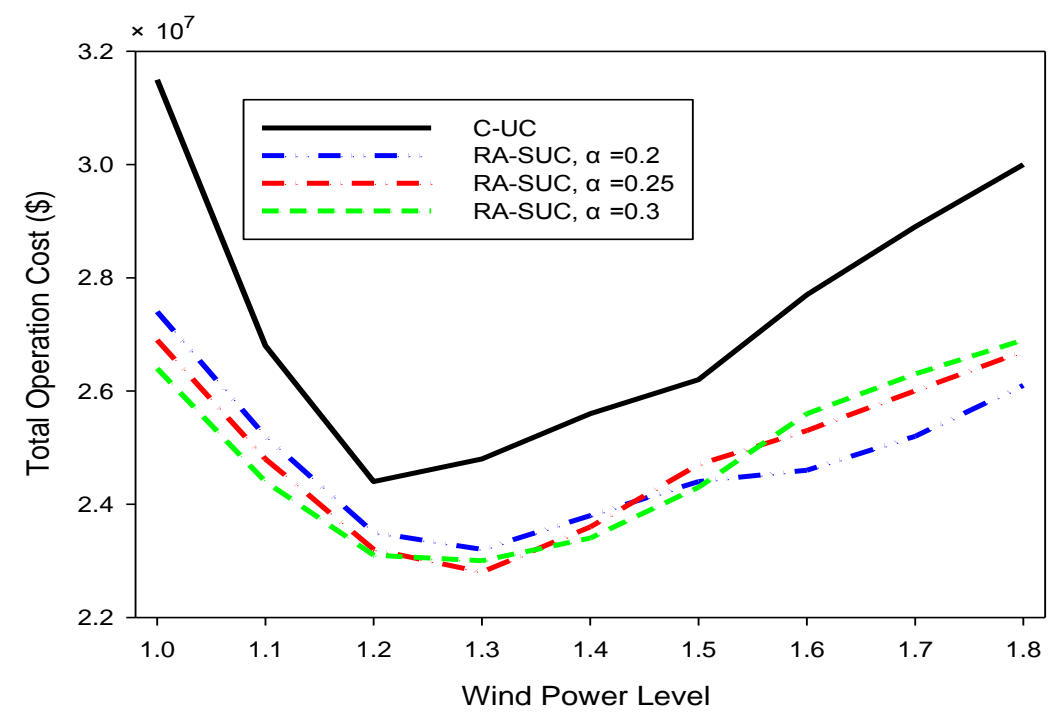

Figure 3. Comparison of total operation cost versus wind power level. C-UC: conventional UC; RA-SUC: risk-averse stochastic unit commitment.

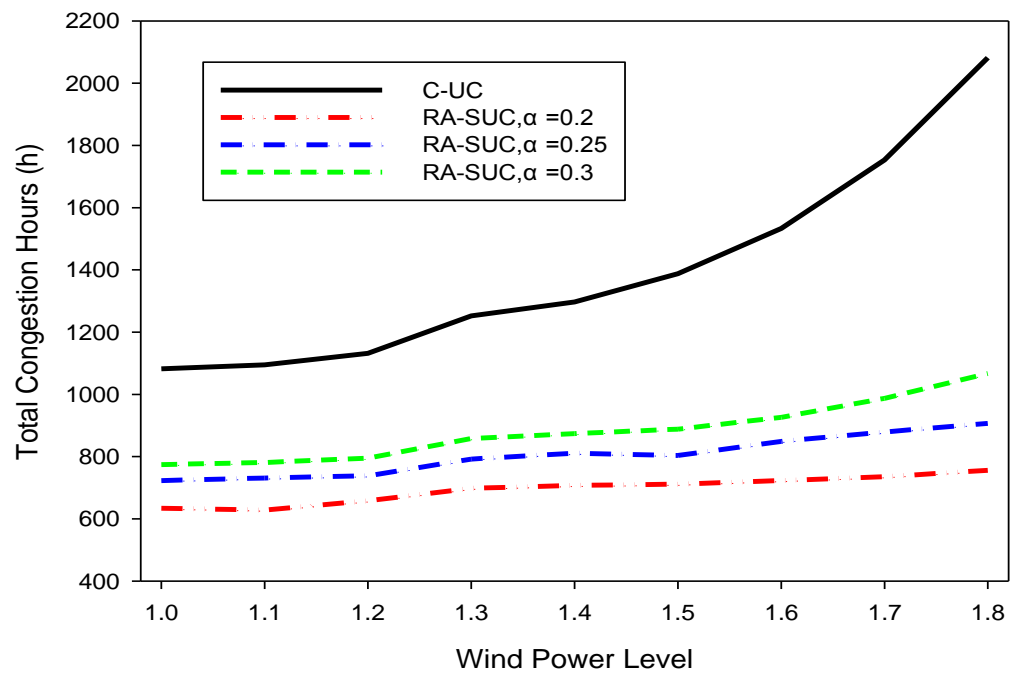

Figure 4. Comparison of total congestion hours versus wind power level.

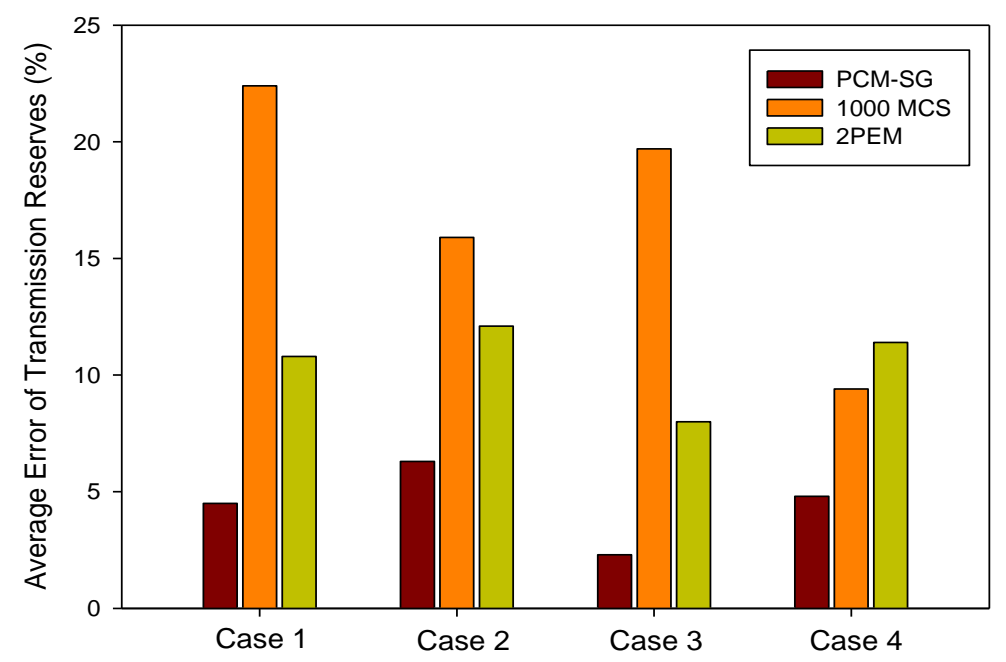

Figure 5. Average percentage error of real-time transmission reserve requirements. PCM-SG: probabilistic collocation method with sparse grids. 


\section{Conclusions}

In this paper, we present a congestion risk-averse stochastic unit commitment model with transmission reserves to hedge against uncertainty and variability induced by wind generation. The proposed model utilizes the transmission reserves in the real-time operation in response to the uncertainties imposed by the spatially-correlated wind power forecast error. By using a high-dimensional probabilistic collocation method in a real-time redispatch formulation, the transmission reserve requirements are quantified in a systematic manner with relatively small computational effort and high accuracy. Specifically, the acquired transmission reserves, which are characterized by the statistical features of line flows, are adaptive to the current operating conditions. Numerical results and analysis on the IEEE 57-bus system demonstrate that the proposed method could potentially reduce the total operation cost by diminishing the congestion risk and reserve undeliverability, as compared to the conventional UC. Moreover, the reduction in cost and congestion hours under different risk and wind power levels confirms the robustness and effectiveness of the proposed method in the enhancement of wind power utilization and system reliability.

Author Contributions: Conceptualization, Y.H., and Q.X.; Methodology, Y.H.; Software, Y.H.; Validation, Y.H., Q.X., and G.L.; Investigation, G.L.; Resources, Q.X.; Data Curation, G.L.; Writing-Original Draft Preparation, Y.H.; Writing-Review \& Editing, Q.X. and G.L..; Supervision, Q.X., and G.L.; Project Administration, Y.H.; Funding Acquisition, Y.H., and Q.X.

Funding: This research was funded by the Fundamental Research Funds for the Central Universities (2242016K41064), Scientific Research Foundation of Graduate School of Southeast University and Postgraduate Research \& Practice Innovation Program of Jiangsu Province, the National Science Foundation (DMS-1555072 and DMS-1736364) and the National Natural Science Foundation of China (grant 51728601).

Acknowledgments: We would like to thank Sajjad Abedi from Purdue University for his kind discussion and valuable suggestions on this work. Further, we are thankful to State Grid Electric Power Research Institute for the availability of wind power forecast and measurement data.

Conflicts of Interest: The authors declare no conflict of interest.

\section{Acronyms}

$\begin{array}{ll}\text { NWP } & \text { numerical weather prediction } \\ \text { ESS } & \text { energy storage systems } \\ \text { DR } & \text { demand response } \\ \text { FACTS } & \text { flexible AC transmission systems } \\ \text { UC } & \text { unit commitment } \\ \text { ED } & \text { economic dispatch } \\ \text { C-UC } & \text { conventional unit commitment } \\ \text { SUC } & \text { stochastic unit commitment } \\ \text { RA-SUC } & \text { risk-averse stochastic unit commitment } \\ \text { MC } & \text { Monte Carlo } \\ \text { MCS } & \text { Monte Carlo simulation } \\ \text { PCM } & \text { probabilistic collocation method } \\ \text { PCM-SG } & \text { probabilistic collocation method with sparse grids } \\ \text { MIP } & \text { mixed integer programming } \\ \text { PDF } & \text { probability density function } \\ \text { CDF } & \text { cumulative probability function } \\ \text { VOWC } & \text { Value of Wind Curtailment } \\ \text { VOLL } & \text { Value of Lost Load } \\ \text { P-OPF } & \text { probabilistic optimal power flow } \\ \text { CP } & \text { collocation point } \\ \text { PEM } & \text { point estimate method } \\ \text { 2PEM } & \text { two-point estimate method } \\ \text { GQ } & \text { Gaussian quadrature } \\ & \end{array}$




\section{References}

1. Hernández-Escobedo, Q.; Manzano-Agugliaro, F.; Zapata-Sierra, A. The wind power of Mexico. Renew. Sustain. Energy Rev. 2010, 14, 2830-2840. [CrossRef]

2. Montoya, F.G.; Manzano-Agugliaro, F.; López-Márquez, S.; Hernández-Escobedo, Q.; Gil, C. Wind turbine selection for wind farm layout using multi-objective evolutionary algorithms. Expert Syst. Appl. 2014, 41, 6585-6595. [CrossRef]

3. Dong, L.; Wang, L.; Khahro, S.F.; Gao, S.; Liao, X. Wind power day-ahead prediction with cluster analysis of NWP. Renew. Sustain. Energy Rev. 2016, 60, 1206-1212. [CrossRef]

4. Andrade, J.R.; Bessa, R.J. Improving renewable energy forecasting with a grid of numerical weather predictions. IEEE Trans. Sustain. Energy 2017, 8, 1571-1580. [CrossRef]

5. Drew, D.R.; Cannon, D.J.; Barlow, J.F.; Coker, P.J.; Frame, T.H.A. The importance of forecasting regional wind power ramping: A case study for the UK. Renew. Energy 2017, 114, 1201-1208. [CrossRef]

6. Pappala, V.S.; Erlich, I.; Rohrig, K.; Dobschinski, J. A stochastic model for the optimal operation of a wind-thermal power system. IEEE Trans. Power Syst. 2009, 24, 940-950. [CrossRef]

7. Choi, J.W.; Kim, M.K. Multi-Objective Optimization of Voltage-Stability Based on Congestion Management for Integrating Wind Power into the Electricity Market. Appl. Sci. 2017, 7, 573. [CrossRef]

8. Bludszuweit, H.; Domínguez-Navarro, J.A.; Llombart, A. Statistical analysis of wind power forecast error. IEEE Trans. Power Syst. 2008, 23, 983-991. [CrossRef]

9. Huang, Y.; Xu, Q.; Jiang, X.; Tong, Z.; Yang, Y. Modelling Correlated Forecast Error for Wind Power in Probabilistic Load Flow. Elektron. Elektrotech. 2017, 23, 61-66. [CrossRef]

10. Pinson, P.; Kariniotakis, G. Conditional prediction intervals of wind power generation. IEEE Trans. Power Syst. 2010, 25, 1845-1856. [CrossRef]

11. Lan, H.; Yin, H.; Wen, S.; Hong, Y.Y.; Yu, D.C.; Zhang, L. Electrical Energy Forecasting and Optimal Allocation of ESS in a Hybrid Wind-Diesel Power System. Appl. Sci. 2017, 7, 155. [CrossRef]

12. Aghaei, J.; Alizadeh, M.I.; Siano, P.; Heidari, A. Contribution of emergency demand response programs in power system reliability. Energy 2016, 103, 688-696. [CrossRef]

13. Nasri, A.; Conejo, A.J.; Kazempour, S.J.; Ghandhari, M. Minimizing wind power spillage using an OPF with FACTS devices. IEEE Trans. Power Syst. 2014, 29, 2150-2159. [CrossRef]

14. Li, N.; Hedman, K.W. Economic assessment of energy storage in systems with high levels of renewable resources. IEEE Trans. Sustain. Energy 2015, 6, 1103-1111. [CrossRef]

15. Henneaux, P.; Kirschen, D.S. Probabilistic security analysis of optimal transmission switching. IEEE Trans. Power Syst. 2016, 31, 508-517. [CrossRef]

16. Moshari, A.; Ebrahimi, A.; Fotuhi-Firuzabad, M. Short-term impacts of DR programs on reliability of wind integrated power systems considering demand-side uncertainties. IEEE Trans. Power Syst. 2016, 31, 2481-2490. [CrossRef]

17. Hreinsson, K.; Vrakopoulou, M.; Andersson, G. Stochastic security constrained unit commitment and non-spinning reserve allocation with performance guarantees. Int. J. Electr. Power. 2015, 72, $109-115$. [CrossRef]

18. Lee, T.Y. Optimal spinning reserve for a wind-thermal power system using EIPSO. IEEE Trans. Power Syst. 2007, 22, 1612-1621. [CrossRef]

19. De, V.K.; Driesen, J. Dynamic operating reserve strategies for wind power integration. IET Renew. Power Gen. 2014, 8, 598-610.

20. Papavasiliou, A.; Oren, S.S.; Rountree, B. Applying high performance computing to transmission-constrained stochastic unit commitment for renewable energy integration. IEEE Trans. Power Syst. 2015, 30, 1109-1120. [CrossRef]

21. Bertsimas, D.; Litvinov, E.; Sun, X.A.; Zhao, J.; Zheng, T. Adaptive robust optimization for the security constrained unit commitment problem. IEEE Trans. Power Syst. 2013, 28, 52-63. [CrossRef]

22. Xu, M.; Zhuan, X. Optimal planning for wind power capacity in an electric power system. Renew. Energy 2013, 53, 280-286. [CrossRef]

23. Morshed, M.J.; Hmida, J.B.; Fekih, A. A probabilistic multi-objective approach for power flow optimization in hybrid wind-PV-PEV systems. Appl. Energy. 2018, 211, 1136-1149. [CrossRef] 
24. Morshed, M.J.; Asgharpour, A. Hybrid imperialist competitive-sequential quadratic programming (HIC-SQP) algorithm for solving economic load dispatch with incorporating stochastic wind power: A comparative study on heuristic optimization techniques. Energy Convers. Manage. 2014, 84, 30-40. [CrossRef]

25. Wang, F.; Hedman, K.W. Dynamic reserve zones for day-ahead unit commitment with renewable resources. IEEE Trans. Power Syst. 2015, 30, 612-620. [CrossRef]

26. Ye, H.; Ge, Y.; Shahidehpour, M.; Li, Z. Uncertainty marginal price, transmission reserve, and day-ahead market clearing with robust unit commitment. IEEE Trans. Power Syst. 2017, 32, 1782-1795. [CrossRef]

27. Zhang, Z.S.; Sun, Y.Z.; Gao, D.W.; Lin, J.; Cheng, L. A versatile probability distribution model for wind power forecast errors and its application in economic dispatch. IEEE Trans. Power Syst. 2013, 28, 3114-3125. [CrossRef]

28. Huang, Y.; Xu, Q.; Yang, Y.; Jiang, X.Q. Numerical method for probabilistic load flow computation with multiple correlated random variables. IET Renew. Power Gen. 2018, 12, 1295-1303. [CrossRef]

29. Hashimoto, T. A method for solving optimal control problems subject to probabilistic affine state constraints for linear discrete-time uncertain systems. Int. J. Mech. Prod. Eng. 2015, 3, 6-10.

30. Jiang, R.; Guan, Y.; Watson, J.P. Risk-averse stochastic unit commitment with incomplete information. IIE Trans. 2016, 48, 838-854. [CrossRef]

31. Huang, Y.; Zheng, Q.P.; Wang, J. Two-stage stochastic unit commitment model including non-generation resources with conditional value-at-risk constraints. Electr. Power Syst. Res. 2014, 116, 427-438. [CrossRef]

32. Gazijahani, F.S.; Salehi, J. Integrated DR and reconfiguration scheduling for optimal operation of microgrids using Hong's point estimate method. Int. J. Electr. Power Energy Syst. 2018, 99, 481-492. [CrossRef]

33. Zhang, X.F.; Pandey, M.D.; Zhang, Y.M. A numerical method for structural uncertainty response computation. Sci. China Technol. Sci. 2011, 54, 3347-3357. [CrossRef]

34. Advanpix, L L C. Multiprecision Computing Toolbox for Matlab. 2006. Available online: https://www. advanpix.com/ (accessed on 6 July 2016).

35. Lin, G.; Elizondo, M.; Lu, S.; Wan, X.L. Uncertainty Quantification in Dynamic Simulations of Large-scale Power System Models using the High-Order Probabilistic Collocation Method on Sparse Grids. Int. J. Uncert. Quant. 2014, 4, 185-204. [CrossRef]

36. Othman, M.M.; Musirin, I. A novel approach to determine transmission reliability margin using parametric bootstrap technique. Int. J. Electr. Power. 2011, 33, 1666-1674. [CrossRef]

37. NARI. [Online]. Available online: http://www.narigroup.com/ (accessed on 9 May 2018).

38. Christie, R. Power System Test Archive. (University of Washington, 1999)[Online]. Available online: http:/ / www.ee.washington.edu/research/pstca/ (accessed on 18 March 2014).

39. CPLEX, V 12.1. User's Manual for CPLEX. Int. Bus. Mach. Corp. 2009, 46, 157. Available online: ftp: //public.dhe.ibm.com/software/websphere/ilog/docs/optimization/cplex/ps_usrmancplex.pdf (accessed on 10 September 2017).

(C) 2018 by the authors. Licensee MDPI, Basel, Switzerland. This article is an open access article distributed under the terms and conditions of the Creative Commons Attribution (CC BY) license (http:/ / creativecommons.org/licenses/by/4.0/). 